\title{
Crystal structure and non-hydrostatic stress-induced
}

\section{phase transition of urotropine under high pressure}

\author{
Piotr A. Guńka*l, Anna Olejniczak ${ }^{2}$, Samuele Fanetti ${ }^{3,4}$, Roberto Bini ${ }^{3,4,5}$, Ines E. Collings ${ }^{\dagger}$, \\ Volodymyr Svitlyk ${ }^{6}$, Kamil F. Dziubek ${ }^{* 3}$
}

${ }^{1}$ Faculty of Chemistry, Warsaw University of Technology, 00-664 Warszawa, Poland

${ }^{2}$ Faculty of Chemistry, Adam Mickiewicz University, 60-780 Poznań, Poland

${ }^{3}$ LENS, European Laboratory for Nonlinear Spectroscopy, 50019 Sesto Fiorentino, Italy

${ }^{4}$ Dipartimento di Chimica, Università degli Studi di Firenze, 50019 Sesto Fiorentino, Italy

${ }^{5}$ Instituto di Chimica dei Composti Organo-Metallici, CNR-ICCOM, 50019 Sesto Fiorentino, Italy

${ }^{6}$ European Synchrotron Radiation Facility, 38043 Grenoble, France

ABSTRACT 
High-pressure behavior of hexamethylenetetramine (urotropine) was studied in situ using angledispersive single-crystal synchrotron X-ray diffraction (XRD) and Fourier transform infrared absorption (FTIR) spectroscopy. Experiments were conducted in various pressure transmitting media (helium and neon for XRD, nitrogen and $\mathrm{KBr}$ for FTIR experiments) to study the effect of deviatoric stress on phase transformations. Contrary to $\mathrm{As}_{4} \mathrm{O}_{6}$ arsenolite, a material of similar cagelike molecular structure, no pressure-induced helium penetration into the crystal structure was observed. Instead, two pressure-induced structural changes are observed. The first one is suggested by the following occurrences: (i) gradual quenching of the magnitudes of atomic displacement parameters, (ii) diminishing libration contribution to the experimental $\mathrm{C}-\mathrm{N}$ bond length, (iii) discontinuity in calculated IR-active vibrational modes and (iv) asymptotically vanishing discrepancy between the experimental and DFT-calculated unit cell volume. All these features reach a plateau at $\sim 4 \mathrm{GPa}$ and can be attributed to a damping of molecular librations and atomic thermal motion, pointing to the existence of a second-order isostructural phase transition. The second transformation, with an onset at $\sim 12.5 \mathrm{GPa}$ is a first-order phase transition to a tetragonal structure, characterized by sluggish kinetics and considerable hysteresis upon decompression. However, it occurs only in non-hydrostatic conditions, induced by a deviatoric stress in the sample. This behavior finds analogies in similar cubic crystals built of highly symmetric cage-like molecules and may be considered a common feature of such systems. DFT computations were performed to model urotropine equation of state and pressure dependence of vibrational modes. Last but not least, it is worth noting successful Hirshfeld atom refinements, carried out for the incomplete high-pressure diffraction data up to $14 \mathrm{GPa}$, yielded more realistic $\mathrm{C}-\mathrm{H}$ bond lengths than the independent atom model.

\section{INTRODUCTION}


Hexamethylenetetramine (urotropine) is a heterocyclic organic amine with molecules of cagelike structure, composed of interconnected six-membered rings, each in the armchair conformation. Such a spatial atomic arrangement resembles that of carbon atoms in the diamond crystal structure and also many organic (like adamantane) and inorganic (like arsenolite form of arsenic trioxide, $\mathrm{As}_{4} \mathrm{O}_{6}$ ) molecules. ${ }^{1,2}$ Urotropine is an important precursor in the production of high energy density materials, such as hexogen or octogen and is also used as a solid fuel. ${ }^{3}$ While not found in terrestrial nature, urotropine is expected to be present in some comets, as it is an important component of refractory organic residues obtained by warming photolyzed cometary ice analogues. ${ }^{4}$ Importantly, the compression of energetic materials ${ }^{5}$ and studies mimicking conditions in planetary systems aimed at better understanding of physicochemical basis of these phenomena are in the forefront of contemporary research.

Noteworthy, urotropine was the first organic compound whose crystal structure was determined by X-ray diffraction in $1923 .{ }^{6,7}$ Due to the high crystal symmetry and one independent molecule with the center of gravity at the origin of the unit cell, the number of symmetry-independent atoms and hence refined parameters is substantially reduced. Indeed, unlike the vast majority of organic compounds, urotropine crystallizes in a high symmetry space group $I \overline{4} 3 m$. In this respect, it is consistent with the preference of other rigid cage-like organic molecules to pack in high symmetry space groups, e.g. solid adamantane (two ambient-pressure phases, $F m \overline{3} m$ and $P \overline{4} 2{ }_{1} c$ ), ${ }^{8}$ cubane (two ambient pressure phases, $R \overline{3}$ ordered and $R \overline{3}$ or $R 3 m$ disordered), ${ }^{9}$ dodecahedrane $(F m \overline{3}),{ }^{10}$ diamantane (four ambient-pressure phases, $\mathrm{Pa} \overline{3}$ at room temperature) $)^{11,12}$ and also inorganic materials, e.g. $\mathrm{As}_{4} \mathrm{O}_{6}$ arsenolite $(F d \overline{3} m)^{13}$ or isostructural $\mathrm{Sb}_{4} \mathrm{O}_{6}$ senarmontite ${ }^{14}$, both made up from discrete adamantoid molecules. In all these structures, high-symmetry special positions coincide with the center of gravity of cage molecules. Any structural changes associated with 
crystal lattice symmetry lowering according to a group-subgroup relation, and in consequence site symmetry lowering, must be thus coupled to anisotropic distortion of the molecular environment and perturbation of the parent structure. Hence, behavior of solid molecular cage compounds in pressure-temperature space poses an especially intriguing subject to explore. Non-ambient studies of these systems revealed a number of phase transitions, ${ }^{8,9,12-25}$ which can be partially attributed to the non-hydrostatic conditions and stress generated beyond the hydrostatic limits of pressuretransmitting media. ${ }^{26-28}$ Naturally, there are numerous examples of materials with various structure types and dimensionalities that show differences in phase transformation pressures under hydrostatic and non-hydrostatic conditions. ${ }^{29-31}$ In general, shear stress is believed to reduce the nominal transition pressure due to either decrease of the height of the potential barrier or highpressure phase nucleation at new defects generated during plastic deformation, as discussed in a comprehensive review by Levitas. ${ }^{32}$ In spite of these reservations, we decided to distinguish a group of cage compounds, forming molecular crystals with high (cubic or hexagonal) symmetry. Relevant literature data are summarized in Table S1.

In contrast to other cage compounds, reports on phase behavior of urotropine are rather scarce. The fully ordered room-temperature cubic urotropine phase is stable also at low temperature down to $15 \mathrm{~K} \cdot{ }^{22,23}$ A previous high-pressure study of urotropine (experiments carried out without pressure transmitting medium abbreviated as PTM) was based solely on pressure evolution of Raman spectra suggesting two phase transitions around 1.4 GPa and 12.5 GPa, without providing structural information. ${ }^{24}$ Besides, our discovery of pressure-induced helium penetration into single crystals of arsenolite begs the question of whether helium can permeate the crystal of urotropine composed of similar globular molecules. ${ }^{13}$ The suggestion of localized interactions between trapped $\mathrm{He}$ atoms and $\mathrm{As}$ atoms featuring active lone electron pairs in $\mathrm{As}_{4} \mathrm{O}_{6} \cdot 2 \mathrm{He}$ provides 
additional stimulus to seek for similar host-guest complexes of urotropine engaging lone pairs of nitrogen atoms. ${ }^{19}$ Furthermore, due to its structural simplicity and a high crystal symmetry, urotropine seems a suited candidate to attempt Hirshfeld Atom Refinements even for the limited high-pressure data, in order to extract hydrogen positions from experimental X-ray diffraction data. Considering the above, it is therefore the purpose of this communication to investigate thoroughly urotropine behavior at high pressure by combining single-crystal X-ray diffraction structural analysis and infrared absorption spectroscopy. Various PTM were used to evaluate the influence of their non-hydrostaticity on the possible phase transitions. In addition, DFT calculations were carried out to provide accurate description of the molecular structure of urotropine as a function of pressure.

\section{MATERIALS AND METHODS}

\section{X-ray diffraction}

Urotropine was purchased from Sigma Aldrich and single crystals suitable for X-ray diffraction were obtained by sublimation at reduced pressure and freshly used for the investigations. Urotropine single crystals were loaded together with ruby spheres into diamond anvil cells (DACs). Diamond anvils with $600-\mu \mathrm{m}$ culets and stainless-steel gaskets preindented to a thickness of $75 \mu \mathrm{m}$ with $300-\mu \mathrm{m}$ diameter holes were used. Diffraction data were collected with a MAR 165 CCD detector at the ID27 beamline $(\lambda=0.3738 \AA)$ and with a Mar555 flat-panel detector at the ID15B beamline $(\lambda=0.4110 \AA)$ of the European Synchrotron Radiation Facility (Grenoble, France). Pressure was determined by the ruby fluorescence shift method. ${ }^{33,34}$ Detector distance was calibrated using $\mathrm{CeO}_{2}$ and $\mathrm{Si}$ powder standards on ID27 and ID15B beamlines, respectively, whereas other instrument model parameters were determined from single-crystal diffraction experiments on enstatite crystals enclosed in DACs. Two runs with He used as the PTM were 
carried out on both beamlines and one run with Ne used as the PTM only on ID15B. The hydrostatic limits of $\mathrm{He}$ and $\mathrm{Ne}$ are specified in the SI. DACs were rotated from -32 to $+32^{\circ}$ and diffraction images were collected every $1^{\circ}$ with an exposure time of $10 \mathrm{~s}$ at ID27 and every $0.5^{\circ}$ with an exposure time of $1 \mathrm{~s}$ at the ID15B beamline. Additionally, wide scans were taken at some pressures with DACs rotated in the same angular range. These data and single-crystal diffraction were processed and analyzed using Dioptas and CrysAlis ${ }^{\mathrm{PRO}}$ software, respectively. ${ }^{35,36}$ Urotropine crystal structures were refined using SHELXL invoked from within Olex2 suite and analyzed with PLATON program. ${ }^{37-39}$ Hydrogen atoms were placed at calculated positions and were refined as riding atoms. CCDC 1982556-1982593 records contain the supplementary crystallographic data for this paper. These data can be obtained free of charge from the joint CCDC's and FIZ Karlsruhe's service to view and retrieve structures via https://www.ccdc.cam.ac.uk/structures/. The wide scans integrated with Dioptas were analysed using GSAS-II. ${ }^{40}$

Hirshfeld Atom Refinements (HARs) were carried out for the diffraction data collected on ID15B beamline with He used as PTM using the HARt module implemented in Olex2. ${ }^{41-43}$ Def2TZVP basis set and restricted Kohn-Sham Hamiltonian along with BLYP exchange-correlation functional were employed. ${ }^{44,45}$ Clusters of charges around central molecules were not generated. Hydrogen anisotropic displacement parameters were refined while extinction was not accounted for. CCDC 2005102-2005128 records contain the supplementary crystallographic data for successful HARs presented in this article.

\section{DFT computations}

Periodic boundary density functional theory (DFT) computations of urotropine crystal structure and energy under high pressure were carried out using pob-TZVP atom-centered Gaussian-type basis set in CRYSTAL09. ${ }^{46-48}$ Crystal structures of urotropine were optimized under set external 
hydrostatic pressure up to $20 \mathrm{GPa}$ using the B3LYP exchange-correlation functional with the Civalleri's $\mathrm{D}^{*}$ correction accounting for dispersion interactions ${ }^{49} \mathrm{XL}$ grid was used for charge integration in real space and $\Gamma$ centered Monkhorst-Pack grid with a shrinking factor of 8 was used for integration in reciprocal space. SCF was considered converged when energy difference between consecutive steps was smaller than $10^{-8} \mathrm{Ha}$ and the Broyden method was applied to speed up SCF convergence. ${ }^{50}$ Geometry optimizations were performed in internal redundant coordinates until RMS of gradients and displacements were smaller than 0.00003 a.u. and 0.00012 a.u., respectively, and the energy difference between following steps was smaller than $10^{-8}$ Ha. The TOLINTEG parameters were set to "7 77716 ". The same parameters were applied for the calculation of IR-active modes' wavenumbers.

\section{Infrared spectroscopy}

Fourier transform infrared (FTIR) absorption measurements were carried out at the European Laboratory for Non-linear Spectroscopy LENS, using a Bruker-IFS 120HR spectrometer adapted for high-pressure studies. ${ }^{51,52}$ Urotropine was purified by sublimation and ground to powder before the experiment. Membrane DACs equipped with $600 \mu \mathrm{m}$ culet diamonds and stainless-steel gaskets preindented initially to $50 \mu \mathrm{m}$ with a $300 \mu \mathrm{m}$ diameter hole were applied. Dried and finely milled $\mathrm{KBr}$ was packed into the gasket hole forming a pellet and filling the sample chamber. A thin layer of $\mathrm{KBr}$ was subsequently scooped out with a needle tip. Then, the sample was deposited on the top of $\mathrm{KBr}$ substrate, deformed plastically and encapsulated uniformly upon closing the DAC. Using this method, infrared spectra could be obtained with reasonable absorbance without saturation. As $\mathrm{KBr}$ is transparent throughout the entire IR region, it does not contribute to any interfering absorbance bands. The second DAC was prepared likewise, but a top of the sample slab was eventually excavated to a depth of several microns, leaving space between the two-layered 
sandwich pellet and the top diamond. This cavity was then cryogenically loaded with nitrogen, ensuring (quasi)hydrostatic compression over a wider pressure range. In both loadings, ruby spheres were placed in the DACs along with the sample to allow pressure determination with the ruby florescence scale. ${ }^{33,34}$ The hydrostatic limits and phase behavior of $\mathrm{KBr}$ and $\mathrm{N}_{2}$ are given in detail in the SI appendix. The parameters (frequency, width and intensity) of the infrared absorption bands were determined using the Fityk program by fitting Voigt profiles after manual baseline subtraction. ${ }^{53}$

\section{RESULTS}

\section{Structural studies}

Urotropine crystallizes in the cubic space group $I \overline{4} 3 m$ (no. 217) with two molecules exhibiting the symmetry of $\overline{4} 3 m\left(T_{d}\right)$ point group per unit cell, with the molecular center of gravity placed at the $2 a$ Wyckoff position. Urotropine represents a peculiar case where the molecular symmetry is preserved in the site and the factor group (one molecule for primitive cell). The amine undergoes monotonic compression up to 19 GPa both in helium and in neon and the space group symmetry of the crystal is preserved up to the highest pressure (see Figure 1). The $3^{\text {rd }}$ order Vinet equation of state (EoS) fitted to the $(V, p)$ data points collected at ID15B with He used as PTM yields $V_{0}=$ 347.71(13) $\AA^{3}, B_{0}=8.53(16) \mathrm{GPa}$ and $B_{0}{ }^{\prime}=9.29(12)\left(V_{0}\right.$ was not fixed in the fitting procedure and its uncertainty comes from the least square fit). ${ }^{54,55}$ One of the reasons we carried out the experiments was to determine whether He permeates urotropine crystal structure in a similar manner to arsenolite. The fact that the unit cells determined for urotropine in $\mathrm{Ne}$ and $\mathrm{He}$ fall on the same EoS line indicates that He does not permeate it. 


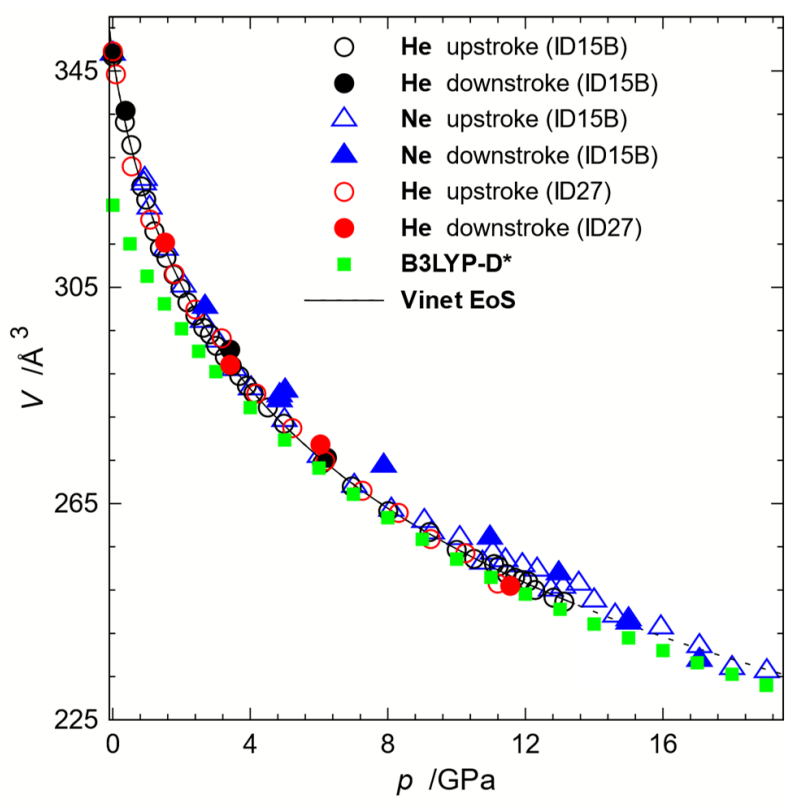

Figure 1. Urotropine unit cell volume plotted as a function of pressure for various experimental runs in He (circles; black and red correspond to data collected at the ID15B and ID27, respectively) and $\mathrm{Ne}$ (triangles) used as PTM. For all the experimental data, the uncertainties on volumes and pressures are smaller than the symbol size. The experimental points are compared to DFT results denoted B3LYP-D* (small green filled squares). Markers are empty and filled for pressure increase and decrease, respectively. The black line corresponds to a $3^{\text {rd }}$ order Vinet EoS fitted to the data collected at the ID15B beamline with $\mathrm{He}$ as PTM. The fit was performed up to $13 \mathrm{GPa}$ and the dashed line above this pressure serves as a guide for the eye.

The dependence of experimentally determined $\mathrm{C}-\mathrm{N}$ bond length on pressure exhibits a maximum around $3 \mathrm{GPa}$ which is the result of two opposing effects: (1) $\mathrm{C}-\mathrm{N}$ bond shortening and (2) quenching of libration with increasing pressure (see Figure 2 and Figure S1 for the dependence of $\mathrm{C}$ and $\mathrm{N}$ anisotropic displacement parameters (ADPs) on pressure). The second effect may seem counterintuitive, but it is not. Libration quenching with increasing pressure leads to apparent increase in bond lengths at low pressures. This is because at low pressures libration diminishes 
very significantly on pressure increase and its effect of apparent bond shortening falls relatively quickly leading to more realistic bond lengths i.e. longer bonds. At higher pressures some residual libration persists and the apparent bond shortening does not change with pressure anymore (see the differences in uncorrected and corrected bond lengths in Figure 2). $\mathrm{C}-\mathrm{N}$ bond lengths were corrected for libration using the approach proposed by Schomaker and Trueblood and the correction was performed using the PLATON program. ${ }^{39,56}$ The idea of the correction lies in interpreting the ADPs of atoms constituting a molecule in terms of anisotropic translational and rotational oscillations of the molecule. ${ }^{57}$ The effect of these angular oscillations is that the maxima of electron density appear to be closer to the center of rotations than they really are i.e. bonds seem to be shorter than they are. Cruickshank derived a formula to correct bond lengths for this effect and his formulation was later generalized by Schomaker and Trueblood. ${ }^{58}$ Urotropine $\mathrm{C}-\mathrm{N}$ bond lengths corrected for libration are in excellent agreement with the DFT computed bond lengths (see Figure 2). This, together with the fact that unit cell volumes of urotropine from DFT calculations are underestimated compared to experiment (see Figure 1), indicates two facts: (1) the contribution of phonons below 4 GPa significantly increases unit cell volume at RT compared to DFT-computed volume at $0 \mathrm{~K}$ and (2) dispersion interactions are in this particular case overestimated by the B3LYP-D* model. 


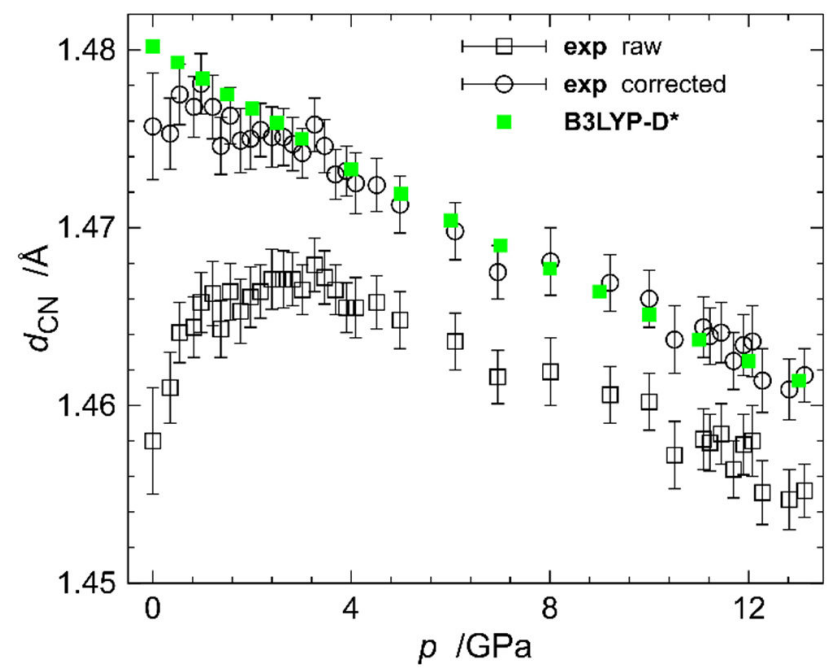

Figure 2. $\mathrm{C}-\mathrm{N}$ bond lengths plotted as a function of pressure for the data collected with He used as PTM at the ID15B beamline. Squares and circles denote experimental bond lengths uncorrected and corrected for libration, respectively. Small green filled squares denote bond lengths obtained from periodic boundary DFT computations.

In the experiment carried out at the ID15B beamline with He used as PTM, we observed a phase transition in urotropine around $14 \mathrm{GPa}$, accompanied by a drop in unit cell volume of $3.30 \AA^{3}$ $(1.30 \%)$. The crystal was completely transformed to a lower symmetry phase, which was accompanied by significant broadening of reflections (see Figure S2 for the raw images of wide scans before and after phase transition). We were able to index this phase using a primitive tetragonal $t P$ unit cell with $a=5.7512(11) \AA$ and $c=7.302(2) \AA$ (at 14.08(12) GPa). This polymorph will be hereafter referred to as $\beta$ while the cubic form will be referred to as $\alpha$. We were unable to solve the crystal structure of the new polymorph based on these data and we carried out Pawley fits of the obtained diffraction patterns to determine unit cell parameters of $\beta$-urotropine up to 20.01(5) GPa in $P 4 / \mathrm{mmm}$ space group (Figure 3). The unit cell shrinks with increasing pressure and three additional reflections located at $3.90^{\circ}, 4.02^{\circ}$ and $13.39^{\circ}$ at $20.01(5) \mathrm{GPa}$ begin to appear which cannot be indexed using this unit cell (see Figure 3). The formation of the $\beta$ 
polymorph is attributed to uniaxial stress exerted on the studied crystal. Its initial size was $\sim 50 \mathrm{x}$ $40 \times 30 \mu \mathrm{m}^{3}$ while the initial gasket thickness was $75 \mu \mathrm{m}$. Due to high He compressibility the gasket shrunk so much that the crystal bridged the diamonds and was subjected to substantial uniaxial stress which resulted in the phase transition. The crystal bridging was evidenced by interference fringes visible on the crystal face that touched the culet (see insets in Figure 3 for crystal photos in DAC under various pressures and Figure $\mathrm{S} 3$ for larger photos). The transition is reversible but there is a significant hysteresis. On pressure release we observed coexistence of two phases down to 9.04(11) GPa and the crystal transformed fully back into the cubic phase at 6.23(7) GPa (see Figure 4 for unit cell parameters and Figure S4 for unit cell volume as a function of pressure plots). The analysis of the orientation matrix and experimental setup geometry allowed us to conclude that the crystal was lying on the culet on the (111) face, indicating it was subjected to uniaxial compression along [111] crystallographic direction. Nonetheless, it did not undergo rhombohedral distortion but tetragonal. We did not observe such transition in the experiment on ID27 beamline because the crystal was not touched by the two diamonds then. Interestingly, wide scans carried out for a crystal pressurized in Ne reveal the coexistence of the tetragonal phase with the body center cubic one beginning from 12.74(8) GPa (see Figure S5). The intensities of reflections coming from the tetragonal phase are weak and increase with pressure indicating a slowly progressing phase transition. It can be concluded that the non-hydrostaticity of neon induces it too. 




Figure 3. Integrated wide scan from the urotropine crystal bridged between two diamonds at 20.01(5) GPa and transformed into the $\beta$ polymorph during the experiment in He PTM at the ID15B beamline. Blue crosses and red line indicate experimental diffraction pattern and Pawley fit of the $\beta$ phase, respectively. The green line is the difference between them plotted on the same scale and moved up to 250 . Black ticks at the bottom denote reflections from $\beta$ polymorph, whereas arrows denote unindexed reflections. Microphotographs of the studied crystal loaded in DAC at various pressures are presented in the inset.

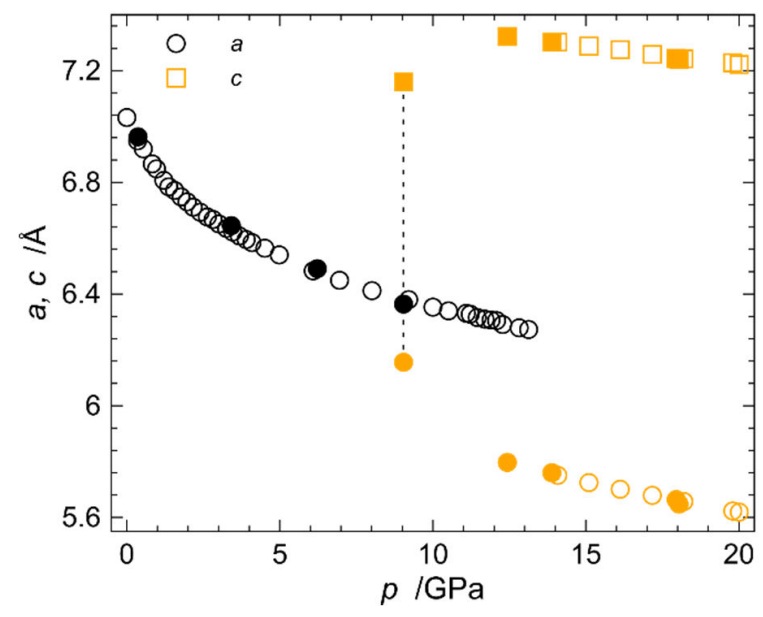


Figure 4. Unit cell parameters plotted as a function of pressure for the $\alpha$ and $\beta$ polymorphs of urotropine. Black and orange symbols correspond to $\alpha$ and $\beta$ unit cells, respectively, whereas open and closed symbols correspond to pressure increase and decrease, respectively.

\section{Hirshfeld atom refinements}

Routine crystal structure refinements employ the independent atom model (IAM) which does not permit for a free refinement of hydrogen atoms positions against X-ray diffraction data due to their weak scattering power. Consequently, they are refined in a constrained manner and the bond lengths involving $\mathrm{H}$ atoms are underestimated e.g. for herein studied crystal all $\mathrm{C}-\mathrm{H}$ bond lengths are set at $0.97 \AA$. It has been recently shown that Hirshfeld atom refinement (HAR) of crystal structures allows one to obtain $\mathrm{C}-\mathrm{H}$ bond lengths within one standard uncertainty of those coming from neutron diffraction measurements and with similar precision. ${ }^{43}$ We attempted HAR for the $\alpha$ polymorph against the diffraction data collected at the ID15B beamline with He used as PTM to check whether it will be successful despite incomplete data sets resulting from the restrictions imposed on the experiment by the use of a DAC (completeness for the maximum attained $2 \theta$ value ranging from $58 \%$ to $78 \%$ ) and whether it is possible to refine $\mathrm{C}-\mathrm{H}$ bond lengths that would be in agreement with ones calculated by DFT. ${ }^{41,42}$ For our data sets, HAR was successful in most cases - only for two pressure points out of 36 did the refinement diverge. In the converged refinements, hydrogen atom ADPs were non positive definite in 7 cases and in three cases they were unphysical ("pancake" ADPs). For the other 26 cases, HAR refinement yielded realistic H atom ADPs. The $\mathrm{C}-\mathrm{N}$ bond lengths were all within one standard uncertainty of the values obtained from conventional refinements. Carbon and nitrogen ADPs did not differ much from the values from conventional spherical atom refinement either. The largest discrepancies for N ADPs were within 3 standard uncertainties and for C ADPs were generally smaller than 3 sigmas and for a few points 
as large as 6 sigmas. The $\mathrm{C}-\mathrm{H}$ bond lengths from HAR oscillate around the $\mathrm{C}-\mathrm{H}$ bond lengths coming from B3LYP-D* computations (see Figure 5). RMSD of experimentally determined C-H bond lengths from DFT calculated ones is $0.038 \AA$ for all successful HARs (only those that yielded physical $\mathrm{H}$ atom ADPs). This is significantly better than IAM for which analogous RMSD equals $0.11 \AA$. It is noteworthy that HARs that yielded unphysical hydrogen ADPs are not trustworthy as the RMSD for $\mathrm{C}-\mathrm{H}$ bond lengths is $0.099 \AA$ in this case - a value that is close to the one corresponding to IAM. Note that the HAR value of the $\mathrm{C}-\mathrm{H}$ bond length of 1.13(3) $\AA$ at $0.34(5)$ GPa is within one standard uncertainty from 1.10(2) $\AA$ which was determined from neutron diffraction experiment on urotropine single crystal carried out at $\sim 0.25 \mathrm{GPa}^{59}$



Figure 5. $\mathrm{C}-\mathrm{H}$ bond lengths plotted as a function of pressure for the $\alpha$ polymorph of urotropine. Big black empty circles and small green filled squares denote bond lengths obtained from HAR and from periodic boundary DFT computations, respectively. Red empty squares indicate bond lengths from HAR in which H ADPs were non positive definite or "pancake-shaped". The purple diamond denotes bond length resulting from refinement against neutron diffraction data.

\section{Infrared absorption spectroscopy}


Urotropine FTIR absorption spectra plotted as a function of pressure are shown in Figure S6. Infrared bands are assigned according to literature data ${ }^{60}$ For the infrared analysis we selected the $\nu_{20}\left(1372.0 \mathrm{~cm}^{-1}\right), \nu_{21}\left(1234.0 \mathrm{~cm}^{-1}\right.$ for TO and $1238.0 \mathrm{~cm}^{-1}$ for LO component), and $v_{24}(674.2$ $\mathrm{cm}^{-1}$ for both TO and LO components) bands because they are in-scale and isolated allowing a good fit in all experimental conditions. All the frequency values refer to ambient conditions. In nitrogen PTM, frequencies of both LO and TO components of the $\mathrm{C}-\mathrm{N}$ bond $\nu_{21}$ stretching mode increase monotonically with pressure without major discontinuities (Figure 6, bottom panel). Near $\sim 12 \mathrm{GPa}$ a new band appears on the lower-frequency side of original $v_{21}$ modes and persists in decompression down to $\sim 8 \mathrm{GPa}$, a point at which the frequencies of $\mathrm{LO}$ and $\mathrm{TO}$ modes revert to their prior values without hysteresis. Compression in $\mathrm{KBr}$, however, shows apparent discontinuities in the slopes of $v_{21} \mathrm{LO}$ and TO branches around $\sim 4$ GPa (Figure 6, top panel). Interestingly, the TO component at higher pressures behaves like the aforementioned new band appearing on compression in $\mathrm{N}_{2}$ environment. We have therefore decided to analyze the line width of all the bands in the upstroke run. Plotting full width at half maximum (FWHM) as a function of pressure demonstrates that the width of $v_{2} \mathrm{LO}$ component in the $\mathrm{KBr} \mathrm{PTM}$ experiment is initially roughly constant, but triples abruptly between 9.6 and $10.0 \mathrm{GPa}$, coinciding with the appearance of the new component in nitrogen above this pressure (Figure S7). It can be therefore assumed that the $v_{21} \mathrm{LO}$ component in $\mathrm{KBr}$ is the convolution of the LO-TO doublet observed in nitrogen, while the lower frequency component is a new band, which appears at lower pressure in the PTM with lower maximum limits of the hydrostatic behavior $(\mathrm{KBr})$. 

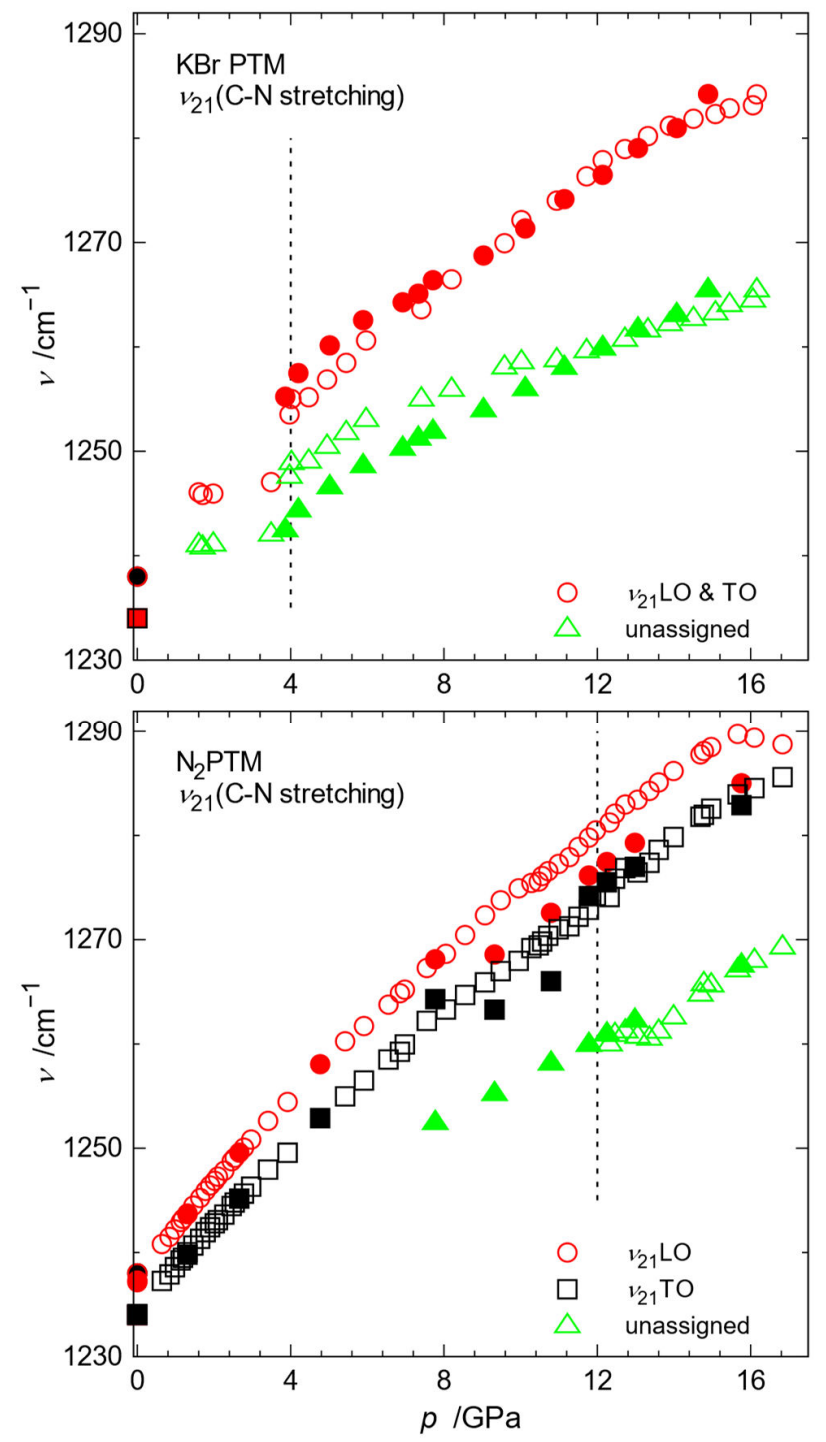

Figure 6. A variation of $\mathrm{LO}$ (red circles) and TO (black squares) components of $v_{21} \mathrm{C}-\mathrm{N}$ stretching mode plotted as a function of pressure in $\mathrm{KBr}$ (top) and $\mathrm{N}_{2}$ (bottom) used as PTM. An additional unassigned band (green triangles) appears in compression in $\mathrm{N}_{2}$ at about $12 \mathrm{GPa}$, persisting in decompression down to $\sim 8 \mathrm{GPa}$. Full symbols refer to downstroke runs. Black circle with red border and red square with black border correspond to literature data at $p=0 .{ }^{60}$ The dashed vertical lines show tentative phase transition pressure, inferred from the changes in the dependence of specific infrared modes. 



Figure 7. Pressure dependence of $v_{20} \mathrm{CH}_{2}$ wagging mode (black squares) plotted as a function of pressure in $\mathrm{KBr}$ (top) and $\mathrm{N}_{2}$ (bottom) PTM. An unassigned soft mode (red circles) appears only in the compression in KBr PTM between 2.0 and 3.5 GPa. Filled symbols refer to downstroke runs. The red square corresponds to literature data at $p=0 .{ }^{60}$ The dashed vertical line shows tentative phase transition pressure, inferred from the changes in the dependence of specific infrared modes. 
The $\mathrm{CH}_{2}$ wagging mode $v_{20}$ follows the same regular continuous pressure dependence in both $\mathrm{N}_{2}$ and $\mathrm{KBr}$ PTM, with a small hysteresis observable only in the sample compressed in $\mathrm{KBr}$. Surprisingly, between 2.0 and $3.5 \mathrm{GPa}$ a soft mode appears in $\mathrm{KBr}$, which is absent in the sample compressed quasihydrostatically in nitrogen (Figure 7). The appearance of the new band is associated with the intensity inversion of the bands in the $\mathrm{C}-\mathrm{H}$ stretching mode regions (Figure S8). It is plausible that the new band might possibly be a Raman mode activated by stress. ${ }^{60}$ Considering that the symmetry of the system does not change from molecule to crystal factor group, thus maintaining the same selection rules of the isolated molecule, lowering of the site symmetry by stress could activate the soft mode mentioned above.

For the $v_{24} \mathrm{C}-\mathrm{N}-\mathrm{C}$ deformation mode, an analogous behavior is observed for both experiments. For the sample in $\mathrm{N}_{2}$ PTM, a new band at a lower frequency side appears at $\sim 12 \mathrm{GPa}$ and persists on decompression down to 7-8 GPa. The frequency of the $v_{24}$ mode remains lower in decompression down to the pressure at which the new band disappears. In case of the sample compressed in $\mathrm{KBr}$, the new band appears at 9-10 $\mathrm{GPa}$ and remains down to $4 \mathrm{GPa}$, i.e. the minimum pressure reached on the decompression run (Figure 8). Since at the atmospheric pressure the $v_{24}$ band is a superposition of TO and LO modes appearing at the same frequency, ${ }^{60}$ it can be supposed that the observation of the new band is actually related to the pressure-induced component splitting.

Wavenumbers of urotropine IR-active vibrational bands were calculated using the B3LYP-D* model and are presented in Figure S9. It is noteworthy that the calculated wavenumbers exhibit discontinuities for all IR-active modes around $4 \mathrm{GPa}$. 


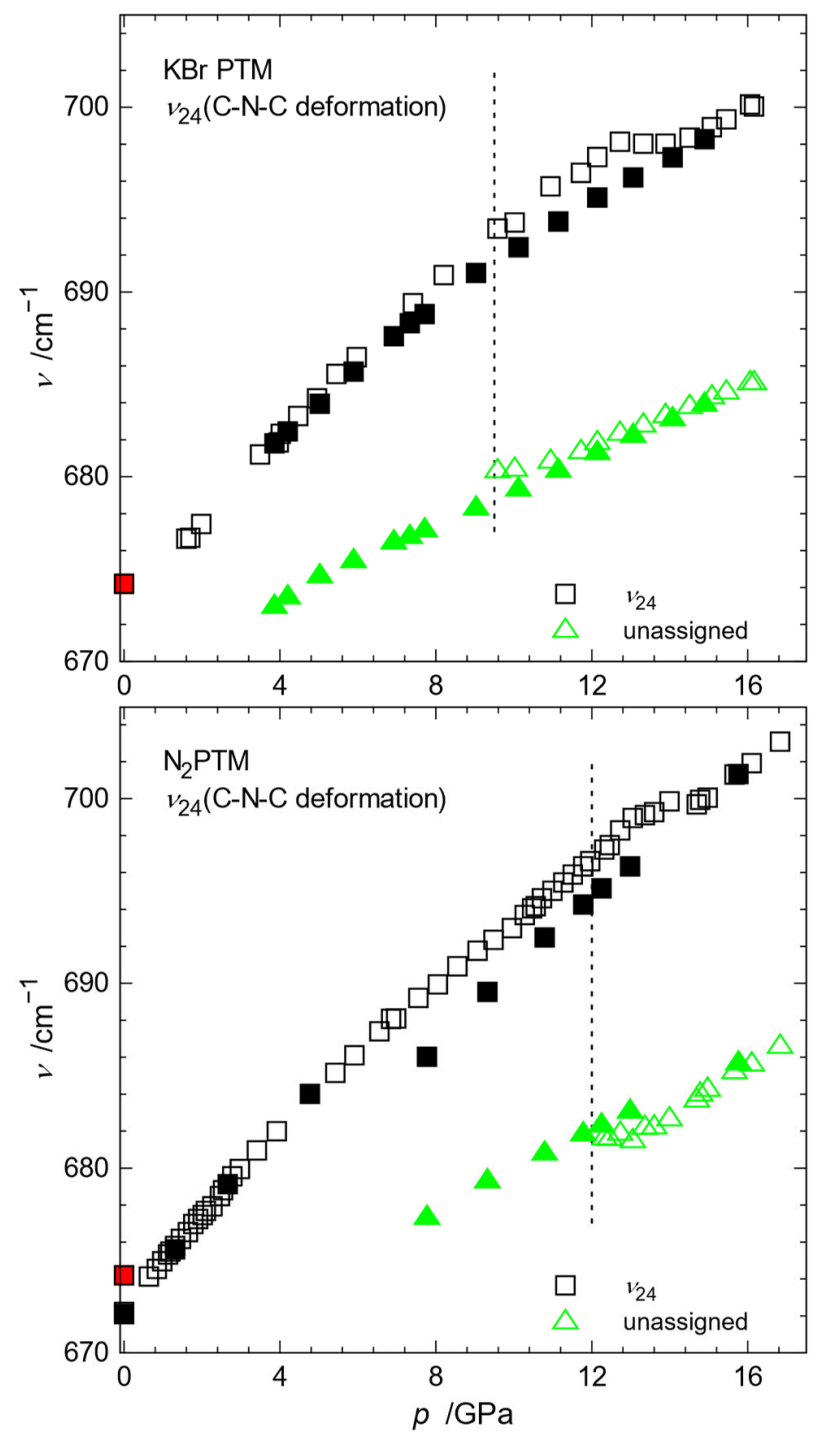

Figure 8. Pressure dependence of $v_{24} \mathrm{C}-\mathrm{N}-\mathrm{C}$ wagging mode (black squares) plotted as a function of pressure together with a new unassigned band that appears on compression (green triangles) in $\mathrm{KBr}$ (top) and $\mathrm{N}_{2}$ (bottom) PTM. Filled symbols refer to downstroke runs. The red square corresponds to literature data at $p=0 .^{60}$ The dashed vertical lines show tentative phase transition pressure, inferred from the changes in the dependence of specific infrared modes. 


\section{DISCUSSION AND CONCLUSIONS}

Investigation of the effects of pressure on crystalline urotropine revealed two structural changes in the pressure behavior. The first one is more subtle and not attributed to any apparent discontinuity in the unit-cell volume. It manifests in the damping of atomic thermal motions evidenced by decrease in the ADP magnitudes of $\mathrm{C}$ and $\mathrm{N}$ atoms with pressure (Figure $\mathrm{S} 1$ ) and suppressed $\mathrm{C}-\mathrm{N}$ bond libration (Figure 2), both completed at $\sim 4 \mathrm{GPa}$, as well as discontinuities in calculated wavenumbers of IR-active modes at this pressure. Remarkably, the static zero-kelvin DFT calculations understate unit-cell volumes up to this critical pressure value. Therefore, it can suggest that the system plausibly undergoes an isostructural second-order transformation of disorder-order type, related to the reduced amplitude of atomic motions or a transition associated with hindering collective molecular rotations about their symmetry elements. Surprisingly, while all these structural features were observed in a diffraction experiment performed under strictly hydrostatic conditions and in calculations where hydrostatic stress was exerted on urotropine, no anomaly was observed in this pressure range in infrared spectra of the urotropine sample in nitrogen PTM. However, in non-hydrostatic PTM (KBr) some abrupt changes become evident around $4 \mathrm{GPa}$, as discontinuities in the slopes of the $\mathrm{C}-\mathrm{N}$ bond $v_{21}$ stretching mode $\mathrm{LO}$ and TO components and appearance of the new soft mode near $v_{20} \mathrm{CH}_{2}$ wagging band. It is pertinent to note here that the literature Raman data collected for the sample without PTM suggest the first transition around $1.4 \mathrm{GPa}^{24}$

The second recorded structural change is an evident first-order phase transition associated with a symmetry lowering from cubic to tetragonal, an abrupt change of lattice parameters and a drop of the unit cell volume beginning at $\sim 12.7 \mathrm{GPa}$ in the experiments carried out at ID15B conducted 
in $\mathrm{Ne}$ and $\sim 14 \mathrm{GPa}$ in $\mathrm{He}$. In the case of the sample in He, the transition onset coincides with the pressure at which the sample begins to bridge the anvils. Meanwhile, in the XRD experiments performed at ID27, no evidence of phase transition could be detected up to 19 GPa which indicates that anvils bridging caused the transition in the case of He experiment at ID15B. FTIR experiments in $\mathrm{N}_{2}$ PTM revealed the appearance of new bands at $\sim 12$ GPa: (1) on the lower-frequency side of original $\mathrm{C}-\mathrm{N}$ bond $v_{21}$ stretching modes and (2) on the lower-frequency side of the $v_{21} \mathrm{C}-\mathrm{N}-\mathrm{C}$ deformation mode. Interestingly, the new band on the lower-frequency side of the $v_{21}$ mode appears also for the sample compressed in $\mathrm{KBr}$ PTM, but with an onset at lower pressure (9-10 GPa). Noteworthy, this pressure values agree well with the literature limit of the second phase transition of $12.5 \mathrm{GPa}$ postulated in the Raman study. ${ }^{24}$ The new bands can be related to the symmetry lowering associated with the transition which causes the activation of new Davydov components. All methods indicate a sluggish character and a considerable hysteresis of this phase change.

Deviatoric stress is known to influence strongly behavior of materials at high pressure, from perturbed determination of lattice parameters to the diverse stability fields of phases. Generally avoided by a careful choice of pressure transmitting medium, sometimes it can provide useful insights and perspectives in solid state chemistry and physics. ${ }^{61}$ Earlier studies of analogous molecules crystallizing in cubic structures at ambient conditions, like diamantane ${ }^{18}$ and arsenolite, ${ }^{20}$ revealed phase transformations occurring only at non-hydrostatic conditions and proceeding with desymmetrization of the crystal lattice. In this respect, urotropine follows the same path, transforming to the tetragonal structure only when subjected to deviatoric stress.

Last but not least, in contrast to arsenolite, we have not observed intercalation of urotropine crystal with helium. Indeed, while the lattice of $\mathrm{As}_{4} \mathrm{O}_{6}$ contains small voids of $\sim 1.8 \AA,{ }^{13}$ a different 
molecular packing pattern in urotropine results in a lack of such cavities (see Figure S10). This, together with the fact that volume reduction is the main driving force of the $\mathrm{As}_{4} \mathrm{O}_{6(\mathrm{~s})}+2 \mathrm{He}_{(\mathrm{s})}=$ $\mathrm{As}_{4} \mathrm{O}_{6} \cdot 2 \mathrm{He}_{(\mathrm{s})}$ process explains why He does not penetrate urotropine. ${ }^{62}$

\section{ASSOCIATED CONTENT}

Supporting Information. The following files are available free of charge.

plots of ADPs, urotropine unit cell volume and bands FWHM as a function of pressure, urotropine crystal microphotographs, integrated diffraction patterns in $\mathrm{Ne}$, evolution of urotropine IR spectra and comparison of experimental and DFT computed bands' wavenumbers (PDF) results of conventional structural refinements (within independent atom model) (CIF) results of HAR (CIF)

\section{AUTHOR INFORMATION}

\section{Corresponding Authors}

*piogun@ch.pw.edu.pl (P.A.G.) dziubek@1ens.unifi.it (K.F.D.)

\section{Present Addresses}

†I.E.C. Center for X-ray Analytics, Empa - Swiss Federal Laboratories for Materials Science and Technology, Überlandstrasse 129, 8600 Dübendorf, Switzerland.

\section{Author Contributions}

All authors have given approval to the final version of the manuscript.

\section{ACKNOWLEDGMENT}


Calculations have been carried out by using resources provided by the Wroclaw Centre for Networking and Supercomputing (http://wcss.pl), grant no. 260. We acknowledge the European Synchrotron Radiation Facility (ESRF) for provision of synchrotron radiation facilities. M. Hanfland and J. Jacobs are acknowledged for gas loading of the DACs for the ID15B beamtime. The Polish contribution to the ESRF budget was financed by the Polish Ministry of Science and High Education - decision number: DIR/WK/2016/19.

\section{REFERENCES}

1 T. C. W. Mak and F.-C. Mok, Inorganic cages related to cubane and adamantane, J. Cryst. Mol. Struct., 1978, 8, 183-191.

2 F. Pertlik, Structure refinement of cubic $\mathrm{As}_{2} \mathrm{O}_{3}$ (arsenolite) with single-crystal data, Czech. J. Phys., 1978, 28, 170-176.

3 P. Roose, K. Eller, E. Henkes, R. Rossbacher and H. Höke, in Ullmann's Encyclopedia of Industrial Chemistry, Wiley-VCH Verlag GmbH \& Co. KGaA, 2015, pp. 1-55.

4 G. Briani, N. Fray, H. Cottin, Y. Benilan, M.-C. Gazeau and S. Perrier, HMT production and sublimation during thermal process of cometary organic analogs. Implications for its detection with the ROSETTA instruments, Icarus, 2013, 226, 541-551.

5 S. M. Peiris and G. J. Piermarini, Eds., Static Compression of Energetic Materials, SpringerVerlag, Berlin Heidelberg, 2008.

6 R. G. Dickinson and A. L. Raymond, The crystal structure of Hexamethylene-tetramine, J. Am. Chem. Soc., 1923, 45, 22-29.

7 H. W. Gonell and H. Mark, Röntgenographische Bestimmung der Strukturformel des Hexamethylentetramins, Z. Phys. Chem., 1923, 107U, 181-218.

8 C. E. Nordman and D. L. Schmitkons, Phase transition and crystal structures of adamantane, Acta Cryst, 1965, 18, 764-767.

9 T. Yildirim, P. M. Gehring, D. A. Neumann, P. E. Eaton and T. Emrick, Unusual Structure, Phase Transition, and Dynamics of Solid Cubane, Phys. Rev. Lett., 1997, 78, 4938-4941.

10C. P. Brock and J. D. Dunitz, Towards a Grammar of Crystal Packing, Chem. Mater., 1994, 6, $1118-1127$.

11 I. L. Karle and J. Karle, The Crystal and Molecular Structure of Congressane, $\mathrm{C}_{14} \mathrm{H}_{20}$, by XRay Diffraction, J. Am. Chem. Soc., 1965, 87, 918-920.

12T. E. Jenkins and A. R. Bates, A Raman study of the three-solid-state phase transition in diamantane, J. Phys. C: Solid State Phys., 1979, 12, 1003-1010.

13P. A. Guńka, K. F. Dziubek, A. Gładysiak, M. Dranka, J. Piechota, M. Hanfland, A. Katrusiak and J. Zachara, Compressed Arsenolite $\mathrm{As}_{4} \mathrm{O}_{6}$ and Its Helium Clathrate $\mathrm{As}_{4} \mathrm{O}_{6} \cdot 2 \mathrm{He}$, Cryst. Growth Des., 2015, 15, 3740-3745.

14Z. Zhao, Q. Zeng, H. Zhang, S. Wang, S. Hirai, Z. Zeng and W. L. Mao, Structural transition and amorphization in compressed $\alpha-\mathrm{Sb}_{2} \mathrm{O}_{3}$, Phys. Rev. B, 2015, 91, 184112.

15 V. Vijayakumar, A. B. Garg, B. K. Godwal and S. K. Sikka, High-pressure phase transitions in adamantane, Chem. Phys. Lett., 2000, 330, 275-280. 
16H.-T. Huang, L. Zhu, M. D. Ward, B. L. Chaloux, R. Hrubiak, A. Epshteyn, J. V. Badding and T. A. Strobel, Surprising Stability of Cubane under Extreme Pressure, J. Phys. Chem. Lett., 2018, 9, 2031-2037.

17 S. H. Bertz, G. A. Kourouklis, A. Jayaraman, G. Lannoye and J. M. Cook, High-pressure Raman studies of triquinacene and dodecahedrane, Can. J. Chem., 1993, 71, 352-357.

18F. Yang, Y. Lin, J. E. P. Dahl, R. M. K. Carlson and W. L. Mao, Deviatoric stress-induced phase transitions in diamantane, J. Chem. Phys., 2014, 141, 154305.

19J. A. Sans, F. J. Manjón, C. Popescu, V. P. Cuenca-Gotor, O. Gomis, A. Muñoz, P. RodríguezHernández, J. Contreras-García, J. Pellicer-Porres, A. L. J. Pereira, D. Santamaría-Pérez and A. Segura, Ordered helium trapping and bonding in compressed arsenolite: Synthesis of $\mathrm{As}_{4} \mathrm{O}_{6} \cdot 2 \mathrm{He}$, Phys. Rev. B, 2016, 93, 054102.

20 A. Grzechnik, Compressibility and Vibrational Modes in Solid $\mathrm{As}_{4} \mathrm{O}_{6}$, J. Solid State Chem., 1999, 144, 416-422.

21 A. L. J. Pereira, L. Gracia, D. Santamaría-Pérez, R. Vilaplana, F. J. Manjón, D. Errandonea, M. Nalin and A. Beltrán, Structural and vibrational study of cubic $\mathrm{Sb}_{2} \mathrm{O}_{3}$ under high pressure, Phys. Rev. B, 2012, 85, 174108 .

22L. N. Becka, D. W. J. Cruickshank and E. G. Cox, The crystal structure of hexam ethylenetetramine I. X -ray studies at 298, 100 and $34^{\circ}$ K, Proc. R. Soc. London, Ser. A, 1963, 273, 435-454.

23 S. P. Kampermann, T. M. Sabine, B. M. Craven and R. K. McMullan, Hexamethylenetetramine: extinction and thermal vibrations from neutron diffraction at six temperatures, Acta Cryst A, 1995, 51, 489-497.

24R. Rao, T. Sakuntala, S. K. Deb, A. P. Roy, V. Vijayakumar, B. K. Godwal and S. K. Sikka, High-pressure Raman scattering studies on hexamethylenetetramine, Chemical Physics Letters, 1999, 313, 749-754.

25 T. Ito, Pressure-induced phase transition in adamantane, Acta Cryst B, 1973, 29, 364-365.

26A. Celeste, F. Borondics and F. Capitani, Hydrostaticity of pressure-transmitting media for high pressure infrared spectroscopy, High Pressure Res., 2019, 39, 608-618.

27 S. Klotz, J.-C. Chervin, P. Munsch and G. L. Marchand, Hydrostatic limits of 11 pressure transmitting media, J. Phys. D, 2009, 42, 075413.

28R. J. Angel, M. Bujak, J. Zhao, G. D. Gatta and S. D. Jacobsen, Effective hydrostatic limits of pressure media for high-pressure crystallographic studies, J. Appl. Crystallogr., 2007, 40, 2632.

29S. Duwal and C.-S. Yoo, Shear-Induced Isostructural Phase Transition and Metallization of Layered Tungsten Disulfide under Nonhydrostatic Compression, J. Phys. Chem. C, 2016, 120, 5101-5107.

30L. Zhang, Y.-H. Li, Y.-Q. Gu and L.-C. Cai, Understanding controversies in the $\alpha-\omega$ and $\omega-\beta$ phase transformations of zirconium from nonhydrostatic thermodynamics, Sci. Rep., 2019, 9, 16889.

31 Y. Zhuang, L. Wu, B. Gao, Z. Cui, H. Gou, D. Zhang, S. Zhu and Q. Hu, Deviatoric stressinduced quasi-reconstructive phase transition in ZnTe, J. Mater. Chem. C, 2020, 8, 3795-3799.

32 V. I. Levitas, High pressure phase transformations revisited, J. Phys.: Condens. Matter, 2018, 30, 163001.

33 H. K. Mao, J. Xu and P. M. Bell, Calibration of the ruby pressure gauge to 800 kbar under quasihydrostatic conditions, J. Geophys. Res.: Solid Earth, 1986, 91, 4673-4676.

34 K. Syassen, Ruby under pressure, High Pressure Res., 2008, 28, 75-126. 
35C. Prescher and V. B. Prakapenka, DIOPTAS: a program for reduction of two-dimensional Xray diffraction data and data exploration, High Pressure Res., 2015, 35, 223-230.

36 CrysAlisPro Software system ver. 171.40.71a, Rigaku OD, Oxford, UK, 2019.

37 G. M. Sheldrick, Crystal structure refinement with SHELXL, Acta Crystallogr. Sect. C, 2015, 71, 3-8.

38O. V. Dolomanov, L. J. Bourhis, R. J. Gildea, J. A. K. Howard and H. Puschmann, OLEX2: a complete structure solution, refinement and analysis program, J. Appl. Crystallogr., 2009, 42, 339-341.

39A. L. Spek, Single-crystal structure validation with the program PLATON, J. Appl. Crystallogr., 2003, 36, 7-13.

40B. H. Toby and R. B. Von Dreele, GSAS-II: the genesis of a modern open-source all purpose crystallography software package, J. Appl. Crystallogr., 2013, 46, 544-549.

41 D. Jayatilaka and B. Dittrich, X-ray structure refinement using aspherical atomic density functions obtained from quantum-mechanical calculations, Acta Crystallogr. Sect. A, 2008, 64, 383-393.

42 S. C. Capelli, H.-B. Bürgi, B. Dittrich, S. Grabowsky and D. Jayatilaka, Hirshfeld atom refinement, IUCrJ, 2014, 1, 361-379.

43 M. Woińska, S. Grabowsky, P. M. Dominiak, K. Woźniak and D. Jayatilaka, Hydrogen atoms can be located accurately and precisely by x-ray crystallography, Science Advances, 2016, 2, e1600192.

44F. Weigend and R. Ahlrichs, Balanced basis sets of split valence, triple zeta valence and quadruple zeta valence quality for H to Rn: Design and assessment of accuracy, Phys. Chem. Chem. Phys., 2005, 7, 3297-3305.

$45 \mathrm{~W}$. Kohn and L. J. Sham, Self-Consistent Equations Including Exchange and Correlation Effects, Phys. Rev., 1965, 140, A1133-A1138.

46R. Dovesi, R. Orlando, B. Civalleri, C. Roetti, V. R. Saunders and C. M. Zicovich-Wilson, CRYSTAL: a computational tool for the ab initio study of the electronic properties of crystals, Z. Kristallogr., 2005, 220, 571-573.

47R. Dovesi, V. R. Saunders, C. Roetti, R. Orlando, C. M. Zicovich-Wilson, B. Pascale, B. Civalleri, K. Doll, N. M. Harrison, I. J. Bush, P. D'Arco and M. Llunell, CRYSTAL09, University of Torino, Torino, 2009.

48 M. F. Peintinger, D. V. Oliveira and T. Bredow, Consistent Gaussian basis sets of triple-zeta valence with polarization quality for solid-state calculations, J. Comput. Chem., 2013, 34, 451459.

49B. Civalleri, C. M. Zicovich-Wilson, L. Valenzano and P. Ugliengo, B3LYP augmented with an empirical dispersion term (B3LYP-D*) as applied to molecular crystals, CrystEngComm, 2008, 10, 405-410.

50D. D. Johnson, Modified Broyden's method for accelerating convergence in self-consistent calculations, Phys. Rev. B, 1988, 38, 12807-12813.

51 R. Bini, R. Ballerini, G. Pratesi and H. J. Jodl, Experimental setup for Fourier transform infrared spectroscopy studies in condensed matter at high pressure and low temperatures, Rev. Sci. Instrum., 1997, 68, 3154-3160.

52F. A. Gorelli, L. Ulivi, M. Santoro and R. Bini, The $\varepsilon$ Phase of Solid Oxygen: Evidence of an O4 Molecule Lattice, Phys. Rev. Lett., 1999, 83, 4093-4096.

53 M. Wojdyr, Fityk: a general-purpose peak fitting program, J. Appl. Crystallogr., 2010, 43, $1126-1128$. 
54P. Vinet, J. Ferrante, J. R. Smith and J. H. Rose, A universal equation of state for solids, J. Phys. C: Solid State Phys., 1986, 19, L467-L473.

55 P. Vinet, J. Ferrante, J. H. Rose and J. R. Smith, Compressibility of solids, J. Geophys. Res.: Solid Earth, 1987, 92, 9319-9325.

56 V. Schomaker and K. N. Trueblood, On the rigid-body motion of molecules in crystals, Acta Crystallogr. Sect. B, 1968, 24, 63-76.

$57 \mathrm{D}$. W. J. Cruickshank, The analysis of the anisotropic thermal motion of molecules in crystals, Acta Crystallogr., 1956, 9, 754-756.

58 D. W. J. Cruickshank, Errors in bond lengths due to rotational oscillations of molecules, Acta Crystallogr., 1956, 9, 757-758.

59 J. Binns, K. V. Kamenev, G. J. McIntyre, S. A. Moggach and S. Parsons, Use of a miniature diamond-anvil cell in high-pressure single-crystal neutron Laue diffraction, IUCrJ, 2016, 3, $168-179$.

$60 \mathrm{~J}$. E. Bertie and M. Solinas, Infrared and Raman spectra and the vibrational assignment of hexamethylenetetramine-h12 and -d12, J. Chem. Phys., 1974, 61, 1666-1677.

61 W. A. Bassett, Deviatoric stress: a nuisance or a gold mine?, J. Phys.: Condens. Matter, 2006, 18, S921-S931.

62 P. A. Guńka, M. Hapka, M. Hanfland, M. Dranka, G. Chałasiński and J. Zachara, How and Why Does Helium Permeate Nonporous Arsenolite Under High Pressure?, ChemPhysChem, 2018, 19, 857-864. 




